\title{
Análise crítica do papel atual da neurorradiologia diagnóstica e intervencionista
}

\author{
Alexandre Varella Giannetti*
}

\section{RESUMO}

A neurorradiologia sofreu grande avanço na última década, abrindo novas perspectivas no diagnóstico e tratamento das doenças cerebrovasculares. Se por um lado, os exames de imagem permitem o diagnóstico por meios não-invasivos, por outro, a via endovascular traz uma opção terapêutica alternativa, isolada ou em associação à microneurocirurgia.

Faz-se uma análise crítica dos artigos mais recentes na literatura, comparando-se os dois métodos terapêuticos, demonstrando o estado atual da neurorradiologia diagnóstica e intervencionista.

\section{PALAVRAS-CHAVE}

Aneurisma intracraniano. Angiografia. Doença cerebrovascular. Má-formação vascular cerebral. Terapia endovascular.

\section{ABSTRACT}

Diagnostic and interventional neuroradiology. A critical analysis and its current status Over the last decade, neuroradiology has presented great progress opening new perspectives in the diagnosis and treatment of cerebrovascular diseases. On one side, the imaging procedures allow the diagnosis by no invasive means, on other hand, the endovascular route brings an alternative therapeutic option, alone or in association to the microneurosurgery.

A critical analysis of the most recent articles of the literature is made, comparing the two therapeutic methods and demonstrating the current status of the diagnostic and interventional neuroradilogy.

\section{KEYWORDS}

Angiography. Cerebral aneurysm. Cerebral AVM. Cerebrovascular disease. Endovascular theraphy.

\section{Introdução histórica}

O desenvolvimento da angiografia cerebral é creditado aos portugueses Antônio de Egaz Muniz e Pedro Almeida Lima pelo trabalho pioneiro realizado em 1926. Naquela época, os autores expunham a carótida cervical, injetavam o contraste e realizavam radiografias simultaneamente. O exame, como inicialmente descrito, passou a ser realizado em todo o mundo até que, em 1953, Sven Ivar Seldinger descreveu a técnica de introdução do cateter por punção e uso de um guia. Tal método permitiu, mais tarde, o uso de uma única via de acesso para a canalização seletiva de várias artérias, reduzindo assim os riscos e tornando o exame mais específico. Nessa mesma época, Leo M. Davidoff desenvolvia o equipamento para troca rápida de filmes, permitindo a seriografia com seis exposições por segundo e conseqüentemente a análise das diversas fases da circulação (arterial, capilar, venosa) em uma única injeção. Em 1961, B. G. Ziedes des Plantes introduziu a técnica de subtração, possibilitando a melhor identificação das estruturas vasculares anteriormente obscurecidas pelas ósseas ${ }^{14}$.

Até então, a neurorradiologia limitava-se basicamente ao campo do diagnóstico. Em 1971, o russo Serbinenko desenvolveu um cateter contendo um balão de látex na extremidade. Mais tarde, em 1978, De Brun, na França, relatava sua experiência inicial com o uso do cateter-balão no tratamento dos aneurismas cerebrais. Esses primeiros ensaios limitavam-se à oclusão da artéria com o balão nos casos de aneurismas que não pudessem ser abordados cirurgicamente, como os intracavernosos, gigantes ou fusiformes. Inicia-se, assim, o segundo grande campo da neurorradiologia, o intervencionista.

Este trabalho originou-se de um parecer solicitado pela Secretaria Municipal de Política Social de Belo Horizonte à Sociedade Mineira de Neurocirurgia, na ocasião em que o autor era o presidente desta última. Tal parecer teve como objetivo fornecer dados concretos para a implantação dos procedimentos endovasculares em neurocirurgia no referido município.

*Ex-Presidente da Sociedade Mineira de Neurocirurgia - Biênio 2000/2001.

Neurocirurgião do Hospital das Clínicas da UFMG e do Hospital Mater Dei, Belo Horizonte, MG. 
Na década de 1980, várias séries foram publicadas usando a técnica de oclusão arterial com o balão, mas já no fim dessa década o interesse passou a ser o da exclusão definitiva do aneurisma, com preservação do fluxo na artéria. Higashida (1990) e Moret (1991) utilizaram balões destacáveis, enquanto Hilal (1989) introduzia pela primeira vez a utilização de molas, mais comumente conhecidas como coils. Em 1991, Guglielmi descreveu pela primeira vez o Guglielmi detachable coil (GDC) o qual sofre uma eletrotrombose antes de ser liberado. A partir daí, esse sistema passou a ser o mais amplamente utilizado, permanecendo até o presente momento ${ }^{40}$.

Toda essa evolução foi acompanhada e permitida pelo desenvolvimento de microcateteres que são hoje subdivididos em dois grupos principais: os dirigidos pela geometria (contendo um microguia) e os dirigidos pelo fluxo sangüíneo. Simultaneamente, inúmeros materiais foram pesquisados para o uso nas embolizações de más-formações vasculares e tumores. São as partículas: PVA geofoam (polyvinyl alcohol foam), colágeno microfibrilar etc. e os agentes líquidos: cianoacrilato, etanol desidratado etc. ${ }^{37}$.

Para a realização da neurorradiologia moderna são necessários equipamentos com alta resolução, contendo técnica de subtração digital e road-mapping. Cateteres e microcateres de diversos tamanhos e modelos, além de diferentes materiais para embolização, devem estar disponíveis para o uso relacionado a cada procedimento específico ${ }^{1,37}$.

Os tópicos a seguir fazem uma análise crítica dos papéis da neurorradiologia no seu estágio atual à luz das publicações mais recentes nas revistas médicas.

\section{Neurorradiologia diagnóstica}

\section{Angiografia cerebral}

A angiografia cerebral permite a visualização de toda a morfologia da vasculatura cerebral, identificando ainda as principais patologias. É considerada o "padrão-ouro" entre os diversos exames existentes. Entretanto, tem dois inconvenientes maiores: é exame invasivo - e por conseqüência tem maior potencial de complicações - e é mais caro.

Alternativas vêm surgindo mais recentemente com a angiorressonância e a angiotomografia. A angiorressonância não utiliza contraste iodado, tem cerca de um terço do custo da angiografia, mas requer muita colaboração do paciene, não pode ser usada em indivíduos com alguns tipos de próteses metálicas ou naqueles que têm claustrofobia. A angiotomografia tem se mostrado de excelente valor na detecção de aneurismas cerebrais com diâmetros superiores a $3 \mathrm{~mm}$, é mais rápida, custa cerca de um quinto do valor da angiografia e requer menor colaboração do paciente em relação aos outros dois exames ${ }^{18,35,36}$.

De uma maneira geral pode-se estipular as seguintes linhas de conduta:

- doença vascular cerebral isquêmica aterosclerótica: angiorressonância que englobe o arco aórtico, carótidas e sistema intracraniano. Ficando a angiografia reservada para os casos duvidosos;

- suspeita de aneurisma familial: angiorressonância ou angiotomografia;

- suspeita de aneurisma como responsável por compressão de nervo craniano: angiotomografia ou angiorressonância;

- hemorragia subaracnóidea espontânea sugestiva de aneurisma cerebral: angiotomografia ou angiografia;

- hemorragia cerebral não-hipertensiva: angiografia;

- má-formação arteriovenosa: angiografia;

- estudo da patência de seios: angiorressonância;

- estudo de pequenos vasos: angiografia.

\section{Testes}

Um estudo angiográfico pode ser acompanhado de testes quando é necessário informação adicional de circulação colateral, seja no planejamento pré-operatório, onde se prevê o sacrifício de uma determinada artéria, seja antes de um procedimento endovascular. Nesses testes, são realizadas a oclusão temporária da artéria com um balão, indução de leve hipotensão, injeções de contraste em outros vasos para estudo de colaterais e avaliações clínica, hemodinâmica e/ou eletroencefalográfica do paciente ${ }^{1,40}$.

Outro teste, necesssário para avaliação de áreas eloqüentes do cérebro (denominado teste de Wada), consiste na injeção de um anestésico de curta duração através de cateterização arterial seletiva ou superseletiva, acompanhada de avaliações clínica e/ou eletroencefalográfica simultâneas.

\section{Neurorradiologia intervencionista}

\section{Tratamento dos aneurismas cerebrais}

Os aneurismas cerebrais podem ser tratados de duas maneiras: microneurocirurgia clássica ou embolização 
endovascular. Em um estudo prospectivo de Koivisto e cols. $^{24}$, foram comparados dois grupos iguais de pacientes que sofreram hemorragia subaracnóidea espontânea por aneurisma cerebral e que foram tratados na fase aguda. A evolução final quanto a morbidade e mortalidade nos dois grupos foi igual ao fim de 3 e 12 meses, inclusive no aspecto neuropsicológico. Do ponto de vista de imagem, o grupo operado apresentou maior número de alterações. Este último aspecto também foi observado no trabalho de Hadjivassiliou e cols. ${ }^{17}$. Ambos concordam que o principal fator prognóstico nesses pacientes está ligado à hemorragia inicial e suas complicações.

Se por um lado, os resultados a curto termo parecem ser semelhantes, nota-se que o efeito da cirurgia a longo prazo é duradouro, pois essa técnica já vem sendo praticada há cerca de 30 anos. Por outro lado, os seguimentos da terapêutica endovascular são inferiores a cinco anos.

Observa-se ainda, na literatura pesquisada, que o tratamento é interrompido durante o procedimento endovascular por dificuldades técnicas em 5\% a 23\% dos casos ${ }^{5,9,26,28}$. Por outro lado, o acesso cirúrgico a certos aneurismas pode ser acompanhado de maior morbidade do que na via endovascular, como nos casos de aneurisma localizado no segmento intracavernoso, na junção vertebrobasilar, no tronco e topo da artéria basilar ${ }^{16}$.

Uma vez que o tratamento do aneurisma cerebral é principalmente profilático no aspecto que visa a sua exclusão da circulação e, conseqüentemente, eliminar o risco de novo sangramento, as questões que se impõem para qualquer proposta terapêutica são: a oclusão foi completa? Caso afirmativo, é ela permanente? Caso negativo, há risco de crescimento do aneurisma residual? Há, por conseqüência, risco de novo sangramento? As séries relatadas na literatura pesquisada tentam responder a essas questões, mas sofrem algumas limitações metodológicas: muitos doentes não são submetidos a angiografias de controle a longo prazo por diversas razões: óbito, instabilidade clínica, perda do contato com a instituição, ou recusa a se submeter a um novo exame. Além disso, as casuísticas publicadas não permitem comparar esses dois tipos de tratamento, pois não são casuísticas equivalentes, existindo diversas variáveis que podem interferir no resultado final, tais como: tamanho, localização e morfologia dos aneurismas, estado clínico do paciente, presença de doenças concomitantes, idade etc. Existe ainda metodologia diferente entre os diversos autores, em especial na definição de colo remanescente, taxa de oclusão subtotal (para alguns $90 \%$, outros $95 \%$ ) ou incompleta. A técnica angiográfica para estudo pósoperatório depende das incidências e principalmente dos equipamentos utilizados (as angiografias das séries cirúrgicas das décadas de 1970 e 1980 não apresentavam as mesmas definições de imagem que os equipamentos atuais empregados nas avaliações dos resultados da terapêutica endovascular). Apesar dessas limitações, algumas comparações e conclusões podem ser tiradas:

Quanto à oclusão total: Thornton e cols. ${ }^{34}$ encontraram na literatura recente (após 1979) seis artigos analisando o resultado avaliado pela angiografia do tratamento cirúrgico de aneurismas cerebrais. Do total de 1.569 aneurismas em 1.370 pacientes, $82(5,2 \%)$ apresentaram enchimento residual na angiografia pósoperatória (variou de 2,3\% a 14\%). Os trabalhos não fizeram distinções nas morfologias dos aneurismas, embora fossem situados nas diversas localizações habituais. Já na série de Sanson e cols. ${ }^{32}$, que estudou apenas aneurismas do topo da artéria basilar, a incidência de aneurisma residual na angiografia pósoperatória de 246 pacientes foi de $6 \%$.

Nas séries de tratamento endovascular, as taxas de oclusão total são em geral inferiores às de tratamento cirúrgico, em torno de $45 \%$ a $63 \%$, $19,26,28,33$. No estudo multicêntrico encomendado pelo FDA (Food and Drug Administration) nos Estados Unidos, que analisou apenas lesões do topo da basilar, dos 150 aneurismas embolizados, em $75 \%$ obteve-se oclusão superior a $90 \%$ na angiografia logo após o procedimento 9 . Entretanto, a morfologia do aneurisma, em especial as suas dimensões, são fatores determinantes na taxa de sucesso. Debrun e cols. ${ }^{7}$ enfatizam que se a relação diâmetro do dome/ diâmetro do colo for superior a 2 e caso o colo seja menor que $5 \mathrm{~mm}$ a chance de oclusão total do aneurisma sobe para $84 \%$. Murayama e cols. ${ }^{28}$, analisando uma série de 120 aneurismas de achado incidental, observaram que, nos casos em que o tamanho do saco foi menor que 10 $\mathrm{mm}$ e do colo inferior a $4 \mathrm{~mm}$, a taxa de oclusão total chegou a $85 \%$. Para aneurismas pequenos com colo maior que $4 \mathrm{~mm}$, grandes e gigantes, as taxas foram, respectivamente, $51 \%$, 44\% e 3\%. Em 1999, Gruber e cols. ${ }^{15}$ publicaram uma pequena casuística de 31 aneurismas maiores que $20 \mathrm{~mm}$ submetidos à embolização com GDC. A oclusão considerada total (>95\%) foi obtida em $69 \%$. Ela foi subtotal ( $90 \%$ a $95 \%$ ) em $13 \%$ e incompleta em $19 \%$. Na tentativa de contornar a dificuldade técnica na embolização de aneurismas cuja razão fundo/ colo seja desfavorável, foi desenvolvida a técnica de remodelagem com balão. No trabalho de Malek e cols. ${ }^{26}$, onde essa razão era de 1,33, nos 17 aneurismas tratados, a taxa média de oclusão foi de $97 \%$. Entretanto, o procedimento não pôde ser completado em 5 pacientes dos 22 da série.

Risco de recanalização após oclusão completa: relatos de casos de aneurismas que se formaram novamente no local onde foi feita clipagem prévia e cuja angiografia pósoperatória demonstrava oclusão satisfatória são encontrados na literatura ${ }^{7}$. Entretanto, são poucos os 
trabalhos que apresentam séries de doentes operados e que se submeteram sistematicamente à angiografia a longo prazo. David e cols. ${ }^{6}$ realizaram o estudo angiográfico com seguimento médio de 4,4 anos em 102 pacientes com 147 aneurismas submetidos à clipagem cirúrgica. Dois aneurismas recorreram $(1,5 \%)$, perfazendo uma taxa anual de recorrência de $0,52 \%$.

Na série de Debrun ${ }^{7}, 4 \%$ dos pacientes que apresentavam exclusão total do aneurisma no fim do procedimento endovascular voltaram a se contrastar no exame subseqüente. $\mathrm{O}$ tamanho da recanalização foi inferior a $5 \%$ do tamanho original do aneurisma e pôde ser conferido tanto no exame de controle de 6 meses quanto no tardio de 2 anos. Já Murayama e cols. ${ }^{28}$ não observaram recanalização em nenhum aneurisma totalmente ocluído em um seguimento médio de 16,3 meses.

Evolução dos aneurismas após oclusões subtotais e incompletas: David e cols. ${ }^{6}$ observaram $12(8,2 \%)$ casos de colos residuais entre 147 aneurismas submetidos à clipagem. Pequena dobra entre o clipe e o vaso teve menor risco de crescimento (25\%) do que em aneurismas cuja clipagem exigiu a reconstrução da parede arterial (75\%). Sanson e cols..$^{32}$ observaram que de 11 aneurismas operados e que apresentaram enchimento residual na angiografia pós-operatória, 4 cresceram e por isso foram reoperados.

Nas séries de tratamento endovascular, a evolução dos colos remanescentes têm sido mais detalhadamente analisada. Murayama e cols. ${ }^{28}$ observaram que do grupo de aneurismas com colos remanescentes que foram seguidos por tempo médio de 16,3 meses, $36 \%$ trombosaram evoluindo para a oclusão total, $32 \%$ permaneceram inalterados e $32 \%$ recanalizaram. Os aneurismas cuja embolização foi incompleta não foram seguidos, sendo submetidos a outro tratamento complementar logo após o endovascular.

Risco de novo sangramento após o tratamento: segundo David e cols. ${ }^{6}$, o risco de sangramento após a clipagem do aneurisma é de $0,26 \%$ ao ano. Uma vez que os autores não obtiveram sangramento no grupo de pacientes cujo aneurisma foi totalmente obliterado, a taxa sobe então para $1,5 \%$ ao ano ( 1 caso entre 12 aneurismas com colos residuais e seguidos por cerca de 4,4 anos).

No trabalho encomendado pelo FDA $^{9}$ sobre aneurismas da artéria basilar submetidos à embolização e seguidos por um ano, a taxa de sangramento após o tratamento foi de 3,6\%. Para aneurismas grandes ou gigantes esta taxa foi de $2,5 \%$ por ano ${ }^{14}$.

Resultado após o tratamento em aneurisma com efeito de massa: em aneurismas que se apresentam com sintomatologia relacionada ao seu efeito de massa, a cirurgia pode promover sua redução, seja por punção e esvaziamento do saco aneurismático seja por sua própria ressecção, ao mesmo tempo em que se exclui o aneurisma da circulação, impedindo a ruptura futura.
Diante da dúvida de se a embolização ao encher o aneurisma não iria reduzir o seu efeito expansivo, 19 pacientes foram avaliados por Malisch e cols. ${ }^{27}$. Esses autores observaram melhora e resolução dos sintomas em $74 \%$ dos casos e piora permanente em 5\% (1 caso). Piora temporária foi notada em três casos, o que também é relatado em casos operados.

\section{Tratamento das más-formações arteriovenosas}

As más-formações arteriovenosas podem ser subdivididas em dois grupos de acordo com a localização: durais (também denominadas de fístulas durais) ou cerebrais.

Em uma meta-análise da literatura de língua inglesa realizada por Lucas e cols. ${ }^{25}$ para o tratamento das másformações arteriovenosas durais, os autores puderam observar que para as lesões do seio cavernoso, o tratamento endovascular é o de eleição, seja por via intra-arterial ou venosa com resultados satisfatórios em $62 \%$ e $78 \%$ respectivamente. Para lesões situadas na fossa anterior, o tratamento cirúrgico foi efetivo em $95 \%$ dos casos. Para lesões do tentório e dos seios transverso e sigmóide o tratamento combinado (embolização e cirurgia) foi mais efetivo que qualquer um deles isoladamente.

No tratamento das más-formações arteriovenosas cerebrais existem três propostas terapêuticas: cirurgia, embolização e radiocirurgia. A escolha depende de algumas variáveis: localização, tamanho e angioarquitetura da lesão, idade e presença de comorbidades. De uma maneira geral, lesões pequenas, superficiais e fora de áreas eloqüentes cerebrais são de indicação cirúrgica, com índices de cura próximo a $100 \%$ e morbidade e mortalidade desprezíveis ${ }^{30}$. Para lesões pequenas (diâmetro inferior a $3 \mathrm{~cm}$ ) e profundas, a escolha recai na radiocirurgia. Esta, entretanto, tem menor taxa de cura $(60 \%$ a $85 \%)$ e leva cerca de 2 anos para se obter a obliteração da lesão, período no qual o doente estaria potencialmente desprotegido. Quanto ao tratamento endovascular, há grande variedade de proposições de materiais a serem utilizados nas embolizações, impedindo maior consenso. De qualquer maneira, Frizzel e Fisher ${ }^{13}$ fizeram a análise de 32 artigos na literatura entre 1960 e 1995 que apresentavam detalhes do tratamento por via endovascular. A soma total de todos os pacientes das séries foi de 1.246. A porcentagem de cura da embolização isoladamente variou de $0 \%$ a $67 \%$, com média de $5 \%$. Em apenas três trabalhos a porcentagem foi superior a $50 \%$. Nas três maiores séries, a taxa de cura foi em torno de $4 \%$. Entretanto, este baixo índice de cura em relação às demais formas de tratamento supramencionadas apresenta uma variável muito importante que justifica 
essa discrepância: os pacientes destas séries de tratamento endovascular apresentavam, em geral, másformações arteriovenosas de grandes volumes. A morbidade temporária do tratamento endovascular oscilou entre $0 \%$ e $50 \%$, com média de $10 \%$, e a morbidade definitiva entre $0 \%$ e $24 \%$, com média de $8 \%$. A mortalidade foi de $0 \%$ a $6 \%$, com média de $1 \%$. Wikholm e cols. ${ }^{38}$, fazendo uma análise de 150 pacientes tratados inicialmente com embolização, também tiveram o baixo índice de $15 \%$ de cura. Entretanto, ao separar os 14 doentes com lesões de diâmetro infeior a $4 \mathrm{~cm}$, este índice subiu para $71 \%$. Se por um lado, a terapêutica endovascular parece ter baixos índices de cura isoladamente, nas lesões de grandes proporções, ela tem tido importante papel como coadjuvante, permitindo a redução da máformação para ulterior tratamento definitivo com a cirurgia ou a radiocirurgia.

\section{Embolização de tumores}

A embolização pré-operatória de lesões, sejam intracranianas, sejam na região da cabeça e pescoço ou mesmo da coluna vertebral, tem se tornado uma prática freqüente, permitindo uma redução na perda sangüínea durante $\mathrm{o}$ ato operatório, facilitando a remoção da lesão, reduzindo o tempo cirúrgico e, conseqüentemente, os riscos da cirurgia. A embolização pré-operatória é tipicamente considerada apropriada em casos de tumores hipervascularizados, como nasoangiofibroma juvenil, paragangliomas, tumores neurogênicos, tumores ósseos primários ou metastáticos e hemangioblastomas ${ }^{1}$. No caso dos meningeomas, o estudo comparativo entre um grupo que foi embolizado no pré-operatório e outro que não o foi, não se observou diferença na dificuldade cirúrgica ou no resultado funcional final ${ }^{3}$.

Algumas vezes, o controle da hemorragia por via endovascular também pode ser necessário como em epistaxe idiopática ou traumática. A injeção de quimioterápico por via intra-arterial para o câncer de cabeça e pescoço é outra alternativa terapêutica que vem se estabelecendo ${ }^{1}$.

\section{Prevenção da isquemia cerebral na doença arterial obstrutiva}

A doença obstrutiva arterial tem na arteriosclerose sua principal etiologia. Entre os sítios de estenose, a bifurcação da artéria carótida comum ao nível do pescoço é o mais freqüente. A origem das artérias no arco aórtico ou nas subclávias e as artérias intracranianas são outros locais de obstrução.

A estenose na bifurcação carotídea tem sua história natural mais amplamente estudada. Seu tratamento, comparando o clínico e o cirúrgico (endarterectomia), já foi avaliado em três trabalhos clássicos e multicêntricos ${ }^{11,12,29}$. Todos eles validam a endarterectomia desde que os resultados do cirurgião sejam acompanhados de no máximo 3\% de complicações isquêmicas para os pacientes assintomáticos e $6 \%$ para os sintomáticos. A angioplastia com colocação de stent surgiu como opção terapêutica, trazendo as vantagens de ser menos invasiva e, por conseqüência, poder ser realizada com anestesia local e, portanto, de menor risco. Não existem, ainda, trabalhos randomizados e multicêntricos como os realizados para a endarterectomia. Entretanto, artigos de grupos isolados têm demonstrado a eficácia do tratamento ${ }^{23}$. Por outro lado, a seleção dos pacientes à terapêutica endovascular não tem sido semelhante aos das séries cirúrgicas, ficando para aquela os doentes que não podem ser submetidos a esta última. Assim, os pacientes das séries de tratamento endovascular são em geral de risco potencialmente maior. Enquanto se aguarda melhor avaliação a longo prazo e com casuísticas maiores, que sejam comparáveis àquelas das endarterectomias, a angioplastia tem suas indicações principais nas seguintes situações: pacientes idosos e com comorbidades que aumentem muito o risco da cirurgia; estenose pósradioterapia; estenose associada à fibrodisplasia; reestenoses; bifurcações carotideanas altas ao nível de $\mathrm{C} 2$; ou presença de outras estenoses intracranianas no território da carótida que se esteja tratando ${ }^{1,20-22}$.

Se por um lado, o tratamento cirúrgico das lesões da bifurcação carotídea é bastante seguro, por outro, nos casos de obstrução na origem das artérias carótidas comuns ou das vertebrais, a cirurgia já se torna mais complexa e com maiores índices de complicações. Nesses casos, a angioplastia com stent mostra-se mais segura e passa a ser o tratamento de primeira escolha. Associa-se a isso o fato de esses tipos de estenose serem mais fibrosas e, por conseqüência, terem menor risco de deslocarem êmbolos durante a dilatação, como ocorre nas bifurcações carotideanas, onde as placas são de ateroma e freqüentemente ulceradas.

Para as estenoses intracranianas, a angioplastia com stent também tem se mostrado eficaz, embora em séries ainda pequenas na literatura. Dessa maneira, fica ainda reservada para situações em que o melhor tratamento conservador tenha falhado ${ }^{1,4}$.

\section{Trombólise intra-arterial na isquemia aguda}

A injeção de substâncias trombolíticas por via intraarterial tem o mesmo objetivo que a infusão de heparina ou trombolíticos por via venosa: abrir o vaso ocluído, 
permitindo a volta da circulação sangüínea na área isquemiada. Em ambas as situações, o fator tempo é determinante, pois o tecido cerebral tolera por muito pouco tempo a interrupção do fluxo sangüíneo. Se por um lado, este é maior fator limitante, outras contraindicações são relatadas: presença de hemorragia intra ou extracerebral, cirurgia recente, punções arterial ou venosa cervical recentes, presença de edema ou apagamento de sulcos na tomografia computadorizada, ausência de circulação colateral na angiografia ou de trombo arterial consistente com os sinais clínicos apresentados pelo paciente. Nota-se assim, que esta terapia fica restrita a casos bastante selecionados ${ }^{1}$.

O principal trabalho que avaliou a injeção de trombolítico intra-arterial, quando esta foi realizada até seis horas do início da isquemia cerebral, demonstrou uma recanalização em cerca de $67 \%$ dos pacientes, o que foi bem superior aos $18 \%$ do grupo-controle. Por outro lado, a incidência de hemorragia intracerebral elevou-se de 5,5\%, no grupo-controle, para $27,8 \%$ no grupo que recebeu a prouroquinase intra-arterial. Clinicamente, o grupo de pacientes submetidos à trombólise intra-arterial teve um benefício relativo de $60 \%$. A mortalidade nos dois grupos foi semelhante ao fim de 90 dias do íctus ${ }^{1}$.

\section{Tratamento endovascular do vasoespasmo cerebral}

Vasoespasmo é a pior e mais comum complicação após hemorragia subaracnóidea espontânea secundária à ruptura de aneurisma cerebral. Ele surge em cerca de $30 \%$ dos pacientes e acarreta morbidade importante ou mortalidade em metade deles.

O tratamento endovascular é tentado geralmente após a falha do tratamento conservador que se baseia principalmente na "terapia dos três Hs": hidratação, hipertensão e hemodiluição ${ }^{1}$. O objetivo consiste em restabelecer o calibre arterial, permitindo o retorno do fluxo sangüíneo cerebral. Tal objetivo pode ser alcançado através da infusão de papaverina, que é menos arriscado, mas de efeito efêmero, ou através da dilatação mecânica usando um balão. Freqüentemente, inicia-se o procedimento com a dilatação química, seguida da mecânica.

Ainda não existe na literatura um trabalho randomizado comparando o tratamento endovascular com o melhor tratamento clínico isoladamente. Os artigos que reportam a angioplastia demonstram resultados bastante favoráveis, com melhora dos sintomas em $61 \%$ a $72 \%$ dos casos em 36 a 72 horas após o procedimento ${ }^{2,10,31}$. Acredita-se que os resultados são tanto mais favoráveis quanto mais precocemente realizados (preferencialmente nas primeiras 24 horas após início dos sintomas). Por outro lado, casos de ruptura arterial durante o procedimento levando ao óbito já foram relatados, demonstrando que o procedimento apresenta riscos $\cos ^{2,10,31}$.

\section{Conclusões}

A neurorradiologia vem sofrendo grande transformação, em especial na última década, podendose notar que ela caminha para deixar de ser menos diagnóstica, com o surgimento de exames menos invasivos - embora ainda tenha grande importância - passando a ter presença mais marcante como opção terapêutica isolada ou em associação a outros tratamentos clínicos ou cirúrgicos. A neurorradiologia intervencionista tem permitido o tratamento de afecções anteriormente consideradas intratáveis, sendo legalmente aceita e reconhecida em todos os países desenvolvidos.

\section{Referências}

1. Accreditation Council on Graduate Medical Education. Standards of Practice: The American Society of International and Therapeutic Neuroradiology: AJNR (suppl): S1-S33, 2001.

2. BEJJANI GK, BANK WO, OLAN WJ, SEKHAR LN: The efficacy and safety of angioplasty for cerebral vasospasm after subarachnoid hemorrahage. Neurosurgery 42:979-87, 1998.

3. BENDSZUS M, RAO G, BURGER R, SCHALLER C, SCHEINEMANN K, WARMUTH-METZ M, HOFMANN E, SCHRAMM J, ROOSEN K, SOLYMOSI L: Is there a benefit of preoperative meningioma embolization? Neurosurgery 47:1306-12, 2000.

4. CONNORS JJ, WOJAK JC: Percutaneous transluminal angioplasty for intracranial atherosclerotic lesions: evolution of technique and short-term results. J Neurosurg 91:415-23, 1999.

5. COTTIER JP, PASCO A, GALLAS S, GABRILLARGUES J, COGNARD C, DROUINEAU J, BRUNEREAU L, HERBRETEAU D: Utility of ballon-assisted Guglielmi Detachable Coiling in the treatment of 49 cerebral aneurysms: A retrospective, multicenter study: AJNR 22:345-51, 2001.

6. DAVID CA, VISHTEH AG, SPETZLER RF, LEMOLE M, LAWTON MT, PARTOVI SL: Late angiographic follow-up of surgically treated aneurysms. J Neurosurg 91:396-401, 1999.

7. DEBRUN GM, ALETICH VA, KEHRLI P, MISRA M, AUSMAN JI, CHARBEL F: Selection of cerebral aneurysms for treatment using Guglielmi Detachable Coils: The preliminary University of Illinois at Chicago experience. Neurosurgery 43:1281-97, 1998. 
8. EBINAK, SUZUKI M, ANDOHA, SAITON K, IWABUCHI $\mathrm{T}$ : Recurrence of cerebral aneurysm after initial neck clipping. Neurosurgery 11:764-8, 1982.

9. ESKRIDGE JM, SONG J: Endovascular embolization of 150 basilar aneurysms with Guglielmi Detachable Coils: results of the Food and Drug Administration Multicenter Clinical Trial. J Neurosurg 89:81-6, 1998.

10. ESKRIDGE JM, MCAULIFFE W, SONG JK, DELIGANIS AV, NEWELL DW, LEWIS DH, MAYBERG MR, WINN HR: Ballon angioplasty for the treatment of vasospasm: Results of first 50 cases. Neurosurgery 42:510-7, 1998

11. The European Carotid Surgery Trialists'collaborative Group: Randomized Trial of Endarterectomy for Recently Symptomatic Carotid Stenosis: Final results of the MRC European Carotid Surgery Trail (ECST). Lancet 351:1379-97, 1998.

12. The Executive Committee for Asymptomatic Carotid Atherosclerosis Study: Endarterectomy for asymptomatic carotid artery stenosis. JAMA 273:1421-8, 1995.

13. FRIZZEL RT, FISHER WS: Cure, morbidity and mortality associated with embolization of brain arteriovenous malformations: A review of 1246 patients in 32 series over a 35 -year period. Neurosurgery 37: 1031-40, 1995.

14. GOBO DJ: Localization techniques: Neuroimaging and electroencephalography. In Greenblat SH, Dagi TF, Epstein MH (eds): A history of neurosurgery in its scientific and professional contexts. Illinois, The American Association of Neurological Surgeons, 1997, pp 228-32.

15. GRUBER A, KILLER M, BAVINSKI G, RICHLING B: Clinical and angiographic results of endovascular coiling treatment of giant and very large intracranial aneurysms: A 7-year, single-center experience. Neurosurgery 45:793-804, 1999.

16. GRUBER DP, ZIMMERMAN GA, TOMSICK TA, VAN LOVEREN HR, LINK MJ, TEW JR JM: A comparison between endovascular and surgical management of basilar artery apex aneurysms. J Neurosurg 90: 86874, 1999.

17. HADJIVASSILIOU M, TOOTH CL, ROMANOWSKI CA, BYRNE J, BATTERSBY RD, OXBURY S, CREWSWELL CS, BURKITT E, STOKES NA, PAUL C, MAYES AR, SAGAR HJ: Aneurysmal SAH: cognitive outcome and structural damage after clipping or coiling. Neurology 26: 1672-7, 2001.

18. HARBAUGH RE, SCHLUSSELBERG DS, JEFFERY R, HAYDEN S, CROMWELL LD, PLUTA D: Threedimensional computerized tomography angiography in the diagnosis of cerebrovascular disease. J Neurosurg 76:408-14, 1992.

19. HOPE JKA, BYRNE JV, MOLYNEUX AJ: Factors influencing succesful angiographic occlusion of aneurysms treated by coil embolization. AJNR 20: 391-9, 1999.

20. HOPKINS LN, LANZINO G, GUTERMAN LR: Treating complex nervous system vascular disorders through a "needle stick": Origins, evolution and future of neuroendovascular therapy. Neurosurgery 48:463-75, 2001.

21. HOROWITZ MB, PURDY PD: The use of stents in the management of neurovascular disease: A review of historical and present status. Neurosurgery 46:133543, 2000

22. Joint Officers of the Congress of Neurological Surgeons and the American Association of Neurological Surgeons: Carotid angioplasty and stent: An alternative to carotid endarterectomy. Neurosurgery 40:344-5, 1997.

23. KELLOGG JX, NESBIT GM, CLARK WM, BARNWELL $S L$ : The role of angioplasty in the treatment of cerebrovascular disease. Neurosurgery 43:549-56, 1998.

24. KOIVISTO T, VANNINEN R, HURSKAINEN H, SAARI T, HERNESNIEMI J, VAPALAHTI M: Outcomes or early endovascular versus surgical treatment of ruptured cerebral aneurysms. A prospective randomized study. Stroke 31:2369-77, 2000.

25. LUCAS CP, ZABRAMSKI JM, SPETZLER RF, JACOBOWITZ R: Treatment for intracranial dural arteriovenous malformations: A meta-analysis from the English language literature. Neurosurgery 40: 1119-32, 1997.

26. MALEK AM, HALBACH VV, PHATOURUS CC, LEMPERT TE, MEYERS PM, DOWD CF, HIGASHIDA RT: Ballon-assist technique for endovascular coil embolization of geometrically difficult intracranial aneurysms. Neurosurgery 46: 1397-407, 2000.

27. MALISCH T, GUGLIELMI G, VIÑUELA F, DUCKWILER G, GOBIN Y, MARTIN N, FRAZEE J, CHMIEL J: Unruptured aneurysms presenting with mass effect symptoms: response to endosaccular treatment with Guglielmi Detachable Coil. Part I: symptoms of cranial nerve dysfunction. J Neurosurg 89:956-61, 1998.

28. MURAYAMA Y, VIÑUELA F, DUCKWILER GR, GOBIN YP, GUGLIELMI G: Embolization of incidental cerebral aneurysms by using the guglielmi detachable coil system. J Neurosurg 90:207-14, 1999.

29. The North American Symptomatic Carotid Endarterectomy Trial: Beneficial effect of carotid endarterectomy in symptomatic patientes with highgrade carotid stenosis. N Engl J Med 325:445-53, 1991.

30. PIK JHT, MORGAN MK: Microsurgery for small arteriovenous malformations of the brain: Results in 110 consecutive patients. Neurosurgery 47:571-7, 2000.

31. POLIN RS, HANSEN CA, GERMAN P, CHADDUCK JB, KASSELL NF: Intra-arterially administered papaverine for treatment of symptomatic cerebral vasospasm. Neurosurgery 42:1256-67, 1998.

32. SAMSON D, HUNT B, KOPITNIK Jr. TA: Current results of the surgical management of aneurysms of the basilar apex. Neurosurgery 44:697-704, 1999.

33. TATESHIMA S, MURAYAMA Y, GOBIN YP, DUCKWILER GR, GUGLIELMI G, VIÑUELA F: Endovascular treatment of basilar tip aneurysms using Guglielmi Deatchable Coils: Anatomic and clinical outcomes in 73 patients from a single institution. Neurosurgery 47:1332-42, 2000.

34. THORNTON J, BASHIR Q, ALETICH VA, DEBRUN GM, AUSMAN JI, CHARBEL FT: What percentage of surgically clipped intracranial aneurysms have residual necks? Neurosurgery 46:1294-300, 2000.

35. VELTHUIS BK, VAN LEEUWEN MS, WITKAMP TD, BOOMSTRA S, RAMOS LMP, RINKEL GJ: CTangiography: Source images and postprocessing techniques in the detection of cerebral aneurysms. AJR 169:1411-17, 1997.

36. VIECO PT, SHUMAN WP, ALSOFROM GF, GROSS CE: Detection of circle of Willis aneurysms in patients with acute subarachnoid hemorrage: A comparison of CT-angiography and digital subtraction angiography. AJR 165:425-30, 1995. 
37. VIÑUELA F, DUCKWILER GR: Endovascular therapy of vascular lesions of central nervous system. In Wilkins $\mathrm{RH}$, Rengachary SS (eds): Neurosurgery New York, McGraw Hill, 1996, pp 631-43.

38. WIKHOLM G, LUNDQVIST C, SVENDSEN P: Embolization of cerebral arteriovenous malformations: Part I - Technique, morphology, and complications. Neurosurgery 39:448-59, 1996

39. WIKHOLM G, LUNDQVIST C, SVENDSEN P: The Goteborg Cohort of Embolized Cerebral Arteriovenous Malformations: A 6-year follow-up. Neurosurgery 49:799-806, 2001.
40. ZUBKOV YN, ALEXANDER L, SMITH RR: Endovascular management of intracranial aneurysms. In Youmans JR (ed): Neurological surgery, 4 ed, Philadelphia, WB Saunders, 1996, pp 1354-71.

Original recebido em fevereiro de 2002

Aceito para publicação em junho de 2002

\section{Endereço para correspondência:}

Alexandre Varella Giannetti

Rua Santa Catarina, 1042 - apt. 201

CEP 30170-080 - Belo Horizonte, $M G$

E-mail:agjg@zaz.com.br 